\title{
Consumo de drogas en estudiantes de un colegio secundario, Corrientes Argentina
}

\author{
Use of drugs among students from a high school, Corrientes - Argentina
}

\author{
María Vallejo, Bárbara Cerda-Aedo, Ismael Morales Ojeda
}

\begin{abstract}
RESUMEN
Objetivo: Identificar los principales factores que llevan a los adolescentes a consumir drogas, analizando la frecuencia de consumo. Metodología: estudio descriptivo y transversal, con una muestra de 124 adolescentes y jóvenes de entre 13 a 21 años, de un colegio secundario público de la provincia de Corrientes-Argentina. Resultados: La experiencia más frecuente de consumo fue con alcohol (87.90\%), luego tabaco (28.22\%) y marihuana (12.09\%) En cuanto a la dimensión "Permisividad Social" y "Accesibilidad a las Sustancias Psicoactivas", el $62.10 \%$ se rodea de amigos que piensan que no está mal emborracharse, a la vez que un $33.06 \%$ siente la necesidad de consumir alcohol o drogas cuando está en un bar, discoteca u otro sitio, considerando el consumo de drogas como algo normal en un $57.26 \%$. En cuanto a la dimensión "Preconceptos", solo el $33.87 \%$ consideró que era difícil para jóvenes como ellos conseguir drogas. Conclusión: En este sentido es necesario brindar el apoyo educativo a la escuela, mediante charlas que permitan hacer frente al consumo de drogas.
\end{abstract}

Palabras clave: Drogas, educación, salud.

\begin{abstract}
Objective: Identify the main factors that lead adolescents to use drugs, analyzing the frequency of use. Methodology: descriptive and cross-sectional study, with a sample of 124 adolescents and young people between 13 and 21 years of age, from a public secondary school in the province of CorrientesArgentina. Results: The most frequent consumption experience was with alcohol (87.90\%), then tobacco $(28.22 \%)$ and marijuana (12.09\%). As for the dimension "Social Permission" and "Accessibility to Psychoactive Substances", $62.10 \%$ He surrounds himself with friends who think that it is not wrong to get drunk, while $33.06 \%$ feel the need to consume alcohol or drugs when they are in a bar, club or other place, considering drug consumption as normal in $57.26 \%$. Regarding the "Preconceptions" dimension, only $33.87 \%$ considered that it was difficult for young people like them to obtain drugs. Conclusion: In this sense, it is necessary to provide educational support to the school, through talks that allow dealing with drug use.
\end{abstract}

Keywords: Drugs, education, health

\footnotetext{
${ }^{1}$ Carrera de Enfermería, Universidad Adventista del Plata, 25 de Mayo 99, Libertador San Martín, Entre Ríos, Argentina.

${ }^{2}$ Psicóloga, Universidad Adventista de Chile, Las Mariposas/Chillán - Chile. 


\section{INTRODUCCIÓN}

La adolescencia no debe entenderse solo como una mera adaptación a las transformaciones corporales, sino como un periodo decisivo del ciclo vital en que se alcanzan tanto la autonomía psicológica como la inserción en el mundo social. (Grusec y Hastings, 2016) El signo característico de este periodo es la necesidad de entrar y formar parte del mundo adulto, cuyos representantes son fundamentalmente los padres. Esta es una etapa decisiva en la consolidación de los estilos de vida, se establecen algunas tendencias comportamentales adquiridas en la infancia y, se incorporan otras nuevas provenientes tanto de los entornos de influencia como del entorno familiar. (Rodrigo et al., 2004) Es necesario tener en cuenta que tanto el compromiso de los padres como el control que ejercen estos, están asociados con una mayor competencia social, autonomía, actitudes positivas hacia la escuela y trabajo, rendimiento académico y nivel de autoestima, disminuyendo la depresión, mala conducta escolar, delincuencia y consumo de drogas. (Allen y Hauser, 1996; Salkovskis, 1985)

La Organización Mundial de la Salud propone los 20 años como edad donde finaliza la adolescencia. Mas en nuestra sociedad muchos jóvenes se ven obligados a seguir siendo socialmente adolescentes hasta edades muy avanzadas, dado que no pueden acceder a las conductas que se consideran propias de los adultos, siguen dependiendoeconómicamentede estos y no tienen verdaderas responsabilidades sociales, laborales y familiares. (Martínez Arias, Fernández Alba y Salgado Ruiz, 2005; Pallarés Gómez y Feixa Pampols, 2000) También se dice que el período de pubertad y de adolescencia se inicia con la combinación del crecimiento rápido del cuerpo y de integración psicosexual y psicosocial. La relación social significativa es la formación de grupo de iguales, por el cual el adolescente busca identificarse con el grupo, ser parte de ello, tener una relación de confianza. (Bordignon, 2005; Erikson, 1970) La adolescencia es un período de apertura al mundo social, por lo que no es infrecuente enc6ontrar dificultades en el área de las habilidades de relación para interactuar en este contexto, de un modo socialmente eficaz. En este sentido, una persona con dificultades para expresar abiertamente sus opiniones o para desenvolverse adecuadamente en su entorno será más vulnerable a la influencia de su grupo. Así, si el consumo de drogas es algo frecuente en este medio, la persona con pocas habilidades sociales, con poca asertividad, tendrá más dificultades para resistirse a la presión de sus compañeros, mientras que sucederá lo contrario en el individuo que posea un buen repertorio de conductas sociales. (Laespada, Iraurgi y Aróstegi, 2004; Luengo, Romero , Gómez , Guerra y Lence,1998)

Las investigaciones también han demostrado que los padres deben participar más en la vida de sus hijos, teniendo conversaciones con ellos acerca del uso de drogas, supervisando sus actividades, conociendo a sus amistades y entendiendo sus problemas e inquietudes personales. (Secades, Fernández y González, 2003)

Una campaña a través de los medios de comunicación bien planificada puede reducir el abuso de sustancias contrarrestando la percepción falsa de que el consumo de drogas es una práctica normal. Las campañas pueden cambiar los comportamientos de riesgo, tales como conducir bajo la influencia del alcohol o, no llevar puesto el cinturón de seguridad. Estas se deben integrar con los programas antidrogas y otras iniciativas de extensión fundamentadas en el hogar, la escuela, los lugares de culto y las organizaciones comunitarias. (Ministerio de Salud/OPS, 2014; Office of National Drug Control Policy, s/f; De Vincenzi y Bareilles; 2011)

De esta manera, el objetivo de este trabajo fue determinar cuáles factores de riesgo estaban presentes en el colegio secundario, determinando cuales eran las drogas más usadas por los estudiantes y el momento $u$ ocasión de consumo de estas.

\section{METODOLOGÍA}

El presente estudio se realizó en un colegio secundario público durante los meses de julio-agosto 2015 en la comunidad de Sauce, Corrientes. La muestra estuvo formada por 124 adolescentes de 13 a 21 años. El diseño de este estudio fue no experimental descriptivo y transversal.

Después de una exhaustiva búsqueda bibliográfica relacionada con instrumentos de medición de consumo de drogas en adolescentes, se seleccionó una encuesta obtenida a partir 
de un estudio realizado en España en el año 2000, el cual fue dirigido por el sociólogo José Navarro Botella (Navarro, 2000) dentro del marco del Plan Municipal contra las drogas del Ayuntamiento de Madrid. Se selecciona el instrumento llamado Factores de Riesgo y Protección para Consumo de Sustancias Psicoactivas debido a que en él se pone especial acento al carácter social del consumo. (Benítez, 2010) Este instrumento evalúa seis dimensiones: malestar emocional, satisfacción con relaciones interpersonales, permisividad social y accesibilidad a las sustancias psicoactivas, espiritualidad, habilidades sociales y de autocontrol, preconceptos y valoración de las sustancias psicoactivas.
Previa solicitud de autorización del responsable de la institución, se procedió a solicitar a los alumnos menores de 18 años que hicieran firmar por sus padres o responsables el consentimiento informado, mientras que los estudiantes de 18 años lo firmaron directamente en el día de la encuesta.

Por último, para analizar los datos se utilizó el paquete estadístico SPSS v.18, con el que se analizaron las características demográficas de la población, las frecuencias de consumo de drogas, su distribución en estudiantes y los factores protectores y de riesgo presentes en la población.

\section{RESULTADOS}

Tabla 1

Frecuencia de consumo de drogas, en un colegio secundario, Corrientes. Argentina.

\begin{tabular}{|c|c|c|c|}
\hline Droga & & Frecuencia & Porcentaje \\
\hline \multirow[t]{6}{*}{ Alcohol } & Nunca & 8 & 6.8 \\
\hline & Diario & 3 & 2.6 \\
\hline & Fines de semana & 69 & 59.0 \\
\hline & una vez por mes & 27 & 23.1 \\
\hline & una vez por año & 10 & 8.5 \\
\hline & Total & 117 & 100.0 \\
\hline \multirow[t]{6}{*}{ Tabaco } & Nunca & 67 & 66.3 \\
\hline & Diario & 18 & 17.8 \\
\hline & Fines de semana & 8 & 7.9 \\
\hline & una vez por mes & 3 & 3.0 \\
\hline & una vez por año & 5 & 5.0 \\
\hline & Total & 101 & 100.0 \\
\hline \multirow[t]{6}{*}{ Marihuana } & Nunca & 78 & 83.9 \\
\hline & Diario & 3 & 3.2 \\
\hline & Fines de semana & 8 & 8.6 \\
\hline & una vez por mes & 1 & 1.1 \\
\hline & una vez por año & 2 & 2.2 \\
\hline & Total & 93 & 100.0 \\
\hline \multirow[t]{5}{*}{ Cocaína } & Nunca & 87 & 94.6 \\
\hline & Diario & 3 & 3.3 \\
\hline & una vez por mes & 1 & 1.1 \\
\hline & una vez por año & 1 & 1.1 \\
\hline & Total & 92 & 100.0 \\
\hline \multirow[t]{4}{*}{ Otros } & Nunca & 66 & 94.3 \\
\hline & Diario & 2 & 2.9 \\
\hline & Fines de semana & 1 & 1.4 \\
\hline & Total & 70 & 100.0 \\
\hline
\end{tabular}


Tabla 2

Creencias y actitudes de los estudiantes del secundario frente al consumo de drogas. Corrientes. Argentina

\begin{tabular}{|c|c|c|c|c|c|}
\hline & \multicolumn{3}{|l|}{ Si } & \multicolumn{2}{|l|}{ No } \\
\hline & fx & $\%$ & & fx & $\%$ \\
\hline \multicolumn{6}{|l|}{ Dimensión Malestar Emocional } \\
\hline Constantemente me siento aburrido & 33 & 26.6 & & 91 & 73.4 \\
\hline Me siento muy afectado por mis problemas & 33 & 26.6 & & 91 & 73.4 \\
\hline Tengo problemas para dormir o me despierto con facilidad & 28 & 22.6 & & 96 & 77.4 \\
\hline Tengo pensamientos repetitivos & 38 & 30.6 & & 86 & 69.4 \\
\hline Tengo dificultad para relajarme & 42 & 33.9 & & 82 & 66.1 \\
\hline Tengo pensamientos que me fastidian & 49 & 39.5 & & 75 & 60.5 \\
\hline Paso fácilmente de la tristeza a la alegría o al revés & 72 & 58.1 & & 52 & 41.9 \\
\hline Me irrito con facilidad & 54 & 43.5 & & 70 & 56.5 \\
\hline Me relajo & 86 & 69.4 & & 38 & 30.6 \\
\hline Me siento angustiado con frecuencia & 33 & 26.6 & & 91 & 73.4 \\
\hline Me tensiono fácilmente & 40 & 32.3 & & 84 & 67.7 \\
\hline Me pongo triste fácilmente & 57 & 46.0 & & 67 & 54.0 \\
\hline \multicolumn{6}{|l|}{ Dimensión Satisfacción con Relaciones Interpersonales } \\
\hline Cuento con alguien para hablar si lo necesito & 99 & 79.8 & & 25 & 20.2 \\
\hline Le agrado a otras personas de mi edad & 101 & 81.5 & & 23 & 18.5 \\
\hline Me siento satisfecho con mis amistades & 111 & 89.5 & & 13 & 10.5 \\
\hline Mis amigos me tienen en cuenta para las actividades que realizan & 107 & 86.3 & & 17 & 13.7 \\
\hline Consigo amigos fácilmente & 86 & 69.4 & & 38 & 30.6 \\
\hline Disfruto compartiendo actividades de recreación con mis amigos & 109 & 87.9 & & 15 & 12.1 \\
\hline $\begin{array}{l}\text { Consulto a alguno de mis padres sobre asuntos de mi vida perso- } \\
\text { nal }\end{array}$ & 66 & 53.2 & & 58 & 46.8 \\
\hline Mis amigos me ayudan a enfrentar mis problemas & 70 & 56.5 & & 54 & 43.5 \\
\hline Hay personas a quienes yo les importo & 115 & 92.7 & & 9 & 7.3 \\
\hline \multicolumn{6}{|l|}{ Dimensión espiritualidad } \\
\hline Tengo fe en un poder más grande que yo & 81 & 65.3 & & 43 & 34.7 \\
\hline No soy una persona religiosa & 75 & 60.5 & & 49 & 39.5 \\
\hline Me apoyo en la religión cuando tengo problemas & 67 & 54.0 & & 57 & 46.0 \\
\hline $\begin{array}{l}\text { Creo que existe una fuerza espiritual que puede ayudarme con mis } \\
\text { problemas }\end{array}$ & 66 & 53.2 & & 58 & 46.8 \\
\hline Me ayuda rezar o meditar & 68 & 54.8 & & 56 & 45.2 \\
\hline $\begin{array}{l}\text { He tenido experiencias espirituales o religiosas importantes en mi } \\
\text { vida }\end{array}$ & 63 & 50.8 & & 61 & 49.2 \\
\hline \multicolumn{6}{|l|}{ Dimensión permisividad social y accesibilidad a las sustancias psicoactivas } \\
\hline Los amigos con los que ando piensan que está mal emborracharse & 47 & 37.9 & 77 & & 62.1 \\
\hline Los amigos con los que ando piensan que está mal drogarse & 90 & 72.6 & 34 & & 27.4 \\
\hline $\begin{array}{l}\text { Siento la necesidad de consumir alcohol o drogas cuando estoy en } \\
\text { un bar. discoteca u otro sitio de ambiente }\end{array}$ & 41 & 33.1 & 83 & & 66.9 \\
\hline $\begin{array}{l}\text { Mi primera opción para una salida con mis amigos es un sitio donde } \\
\text { pueda tomar o consumir drogas }\end{array}$ & 22 & 17.7 & 102 & & 82.3 \\
\hline Me gusta experimentar nuevas sensaciones & 60 & 48.4 & 64 & & 51.6 \\
\hline Tengo algún familiar que bebe hasta emborracharse & 70 & 56.5 & 54 & & 43.5 \\
\hline Consumir drogas es normal entre los jóvenes & 71 & 57.3 & 53 & & 42.7 \\
\hline $\begin{array}{l}\text { Mis amigos incluyen trago. cigarrillo o drogas en las actividades que } \\
\text { realizamos }\end{array}$ & 52 & 41.9 & 72 & & 58.1 \\
\hline
\end{tabular}




\begin{tabular}{|c|c|c|c|c|}
\hline \multicolumn{5}{|l|}{ Dimensión habilidades sociales y de autocontrol } \\
\hline Evito preguntar por temor a hacer el ridículo & 64 & 51.6 & 60 & 48.4 \\
\hline Me siento molesto cuando las cosas no salen a mi manera & 102 & 82.3 & 22 & 17.7 \\
\hline Me preocupo en exceso & 61 & 49.2 & 63 & 50.8 \\
\hline Prefiero ceder para evitar problemas con otras personas & 90 & 72.6 & 34 & 27.4 \\
\hline Me enojo cuando la gente me hace esperar & 72 & 58.1 & 52 & 41.9 \\
\hline Evito herir los sentimientos de alguien a propósito & 90 & 72.6 & 34 & 27.4 \\
\hline Me preocupo cuando rompo una regla & 80 & 64.5 & 44 & 35.5 \\
\hline Pienso mucho antes de actuar & 85 & 68.5 & 39 & 31.5 \\
\hline \multicolumn{5}{|l|}{ Dimensión preconceptos y valoración de las drogas } \\
\hline Para las personas de mi edad es difícil conseguir drogas & 42 & 33.9 & 82 & 66.1 \\
\hline $\begin{array}{l}\text { Para las personas de mi edad es costoso consumir drogas frecuen- } \\
\text { temente }\end{array}$ & 48 & 38.7 & 76 & 61.3 \\
\hline Cuando se bebe en exceso se hace el ridículo & 95 & 76.6 & 29 & 23.4 \\
\hline Las drogas son un problema más grave que el alcohol o el cigarrillo & 103 & 83.1 & 21 & 16.9 \\
\hline
\end{tabular}

El promedio de edad de los 124 estudiantes encuestados fue de $17.29 \pm 2.09$ años, siendo un $65.32 \%$ de sexo femenino. El grupo se conformó principalmente por solteros en un $58.06 \%$ seguido de personas en una relación sentimental formal con un $31.45 \%$. Respecto a la experiencia consumiendo sustancias, los estudiantes usaron alguna vez en el pasado alcohol en un $87.90 \%$ seguido de tabaco $28.22 \%$ y de marihuana $12.09 \%$.

En cuanto al instrumento empleado, en cuanto a la Dimensión Malestar Emocional, en el colegio secundario se pudo apreciar que las preferencias presentes en los estudiantes con puntajes más altos fueron: "Me relajo" 69.35\% seguido de "Paso fácilmente de la tristeza a la alegría o al revés" con $58.06 \%$ y "Me pongo triste fácilmente" $45.97 \%$.

Respecto a la Dimensión Satisfacción con Relaciones Interpersonales, los jóvenes manifestaron principalmente que; "Hay personas a quienes yo les importo" 92.74\% seguido de "Me siento satisfecho con mis amistades" 89.52\%, "Disfruto compartiendo actividades de recreación con mis amigos" $87.90 \%$. Los ítems que menos se presentaron en la vida de los estudiantes fueron; "Consulto a alguno de mis padres sobre asuntos de mi vida personal" $46.77 \%$, "Mis amigos me ayudan a enfrentar problemas" $43.55 \%$, "Consigo amigos fácilmente" $30.65 \%$.

De la Dimensión Espiritualidad, principalmente se presentaron factores como "Tengo fe en un poder más grande que yo" $65.32 \%$, "No soy una persona religiosa" $60.48 \%$, "Me ayuda a rezar o meditar" $54.84 \%$.

En la Dimensión Permisividad Social y Accesibilidad a las Sustancias Psicoactivas destacó la presencia de factores como: "Los amigos con los que ando piensan que está mal drogarse" $72.58 \%$, "Consumir drogas es normal entre los jóvenes" $57.26 \%$, "Tengo algún familiar que bebe hasta emborracharse" $56.45 \%$.

Posteriormente en la Dimensión Habilidades Sociales y de Autocontrol se presentaron los factores, "Me siento molesto cuando las cosas no salen a mi manera" $82.26 \%$, "Prefiero ceder para evitar problemas con otras personas" y "Evito herir los sentimientos de alguien a propósito" ambos con $72.58 \%$. Y "Pienso mucho antes de actuar" $68.55 \%$.

Finalmente, en la Dimensión Preconceptos y Valoración de las Drogas, se dieron factores como: "Las drogas son un problema más grave que el alcohol o el cigarrillo" 83.06\%, "Cuando se bebe en exceso se hace el ridículo" $76.61 \%$, "Para las personas de mi edad es costoso consumir drogas frecuentemente" $38.71 \%$.

\section{DISCUSIÓN}

Si se comparan los datos de este estudio con los encontrados con el consumo de drogas, podemos observar que estos son similares a los arrojados 
por SEDRONAR (Secretaría de Programación para la Prevención de la Drogadicción y la Lucha contra el Narcotráfico, Argentina) es decir que dentro de drogas legalizadas lo que más consumen los adolescentes es el alcohol y dentro de las drogas ilegalizadas, marihuana. (Pedrón, 2008) Algunos estudios reflejan además que la conducta antisocial aparece como un factor de riesgo que está altamente relacionada con las conductas de consumo de alcohol y drogas ilegalizadas en los adolescentes escolares. (López García, 2008) El Ministerio de la Salud de Argentina, por medio del estudio "Algunos datos sobre el consumo de alcohol en Argentina", dio como resultado que el alcohol es la sustancia psicoactiva más consumida por los estudiantes en todo el país, tanto entre los varones como entre las mujeres. (MSAL, 2011) A su vez un $28.22 \%$ de adolescentes de la muestra consume tabaco lo cual resulta preocupante debido a que los adolescentes ya consumen esta droga lícita siendo aún menores de edad. A pesar de todo, en un estudio realizado en 2012, la Encuesta Mundial de Tabaco en Jóvenes del Programa Nacional de Control del Tabaco, arrojó que en Argentina ha descendido el consumo de $24.5 \%$ a $19.6 \%$ en los últimos cinco años, (Ministerio de Salud/OPS, 2014) lo que se puede ver reflejado en la población estudiada, al detectarse que el consumo de tabaco en la opción "Nunca", tenía un $66.34 \%$.

En lo referente a la Dimensión Malestar Emocional, el 58.06\% refirió pasar fácilmente de la tristeza a la alegría o al revés; marcando el ítem me pongo triste fácilmente en un $45.97 \%$. Los adolescentes tienden a tener estados de ánimo cambiantes, y muchas veces tienden en apoyarse en sus amigos porque confían más en sus pares que en sus padres, lo que en algunas ocasiones puede ser de ayuda para prevenir el consumo y otras no. Estos amigos, al tener la misma edad pueden estar viviendo experiencias similares, lo que puede ocasionar que no puedan brindar ayuda en la prevención del consumo de drogas. (Miotto Wright, Gliksman, Khenti y Ferreira Furegato, 2009)

Correspondiente a la Dimensión Satisfacción con Relaciones Interpersonales, se destaca que los adolescentes se sienten muy satisfechos con sus amistades en un $89.52 \%$, además disfrutan actividades de recreación con sus amigos en un $87.90 \%$. Los adolescentes no consultan a sus padres por asuntos de su vida personal en un $46.77 \%$. A medida que los adolescentes van desarrollándose, las oportunidades de conflictos y de incomprensión se incrementan, porque los padres no siempre tienen éxito en el ajuste de su práctica de crianza versus el desarrollo de la autonomía en el adolescente. Debido a esto, los jóvenes prefieren a esta edad hablar de sus problemas emocionales y de sus miedos con sus amigos o pares. (Salkovskis, 1985)

En la Dimensión Espiritualidad, se observa que los adolescentes en un $65.32 \%$ tienen fe en un poder más grande, un $54,84 \%$ piensa que rezar o meditar los ayuda, apoyándose en la religión cuando tiene problemas en un $54,03 \%$. Lo que indica que los adolescentes se apoyan en la religión para solucionar problemas o situaciones difíciles que estén viviendo. Ellos pueden meditar algunas veces cuando se sienten solos y no tienen el apoyo de su familia, amigos o profesores, esto realmente es muy importante en los adolescentes porque les ayuda a escapar de elegir consumir algún tipo de sustancias. Siendo la religiosidad, la creencia en el orden moral, el grado de satisfacción personal respecto a la vida factores de protección del consumo de drogas. (Luengo Martín et al., 1998)

Respecto a la "Dimensión Permisividad Social y Accesibilidad a las Sustancias Psicoactivas", un $62.10 \%$ de los adolescentes de la muestra anda con amigos quienes no creen que está mal emborracharse, teniendo un familiar que bebe hasta emborracharse en un $56.45 \%$; expresando que consumir drogas es normal entre los jóvenes en un $57.26 \%$. Estos datos resultan muy alarmantes porque nos indican que hoy en día en la sociedad y los jóvenes están aceptado el consumo tanto de una droga lícita e ilícita. Para un adolescente, tener un familiar que bebe hasta emborracharse es un factor de riesgo importante, pues puede asimilar que no está mal beber alcohol. La existencia de normas explícitas en la familia que prohíben solamente el uso de drogas ilegales predice un mayor riesgo de consumo de alcohol y tabaco, pues los adolescentes entienden que las drogas legales son menos peligrosas o menos dañinas para la salud al no ser expresamente rechazadas por los padres. (Rodrigo et al., 2004)

La "Dimensión Habilidades sociales y autocontrol", resaltó los ítems: "Me siento molesto cuando las cosas no salen a mi manera" con un $82.26 \%$; "Me enojo cuando la gente me hace esperar" con un $58.06 \%$. En esta dimensión se puede observar que los 
adolescentes tienen muy marcado el enojo y frustración, las personas que tienen relaciones interpersonales conflictivas corren el riesgo de desarrollar insuficientes recursos para afrontar el estrés al distanciarse también de sus familias. Muchas veces estas personas tienden a ver el mundo extremadamente amenazante y hostil, sienten que deben enfrentarlo de manera agresiva o evitarlo a través del escape de la realidad. 5

En la dimensión perteneciente a "Preconceptos y valoración de las drogas", se aprecia que los adolescentes piensan que las drogas son un problema más grave que el alcohol o el cigarrillo

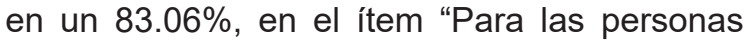
de mi edad es difícil conseguir drogas" un $66.13 \%$ respondió que no. Resaltándose que el conocimiento sobre lo nocivo de las drogas es opuesto al acceso y permisividad social existente.

La mayor o menor adquisición de características personales, sociales, ambientales, familiares, psicológicas, conjuran como factores de riesgo o de protección, facilitando o no la probabilidad de inicio y desarrollo del consumo de drogas, manteniendo el sujeto alejado de esta conducta. (Fernández Hermida y Secades Villa, 2008) No se puede olvidar que los jóvenes sienten la necesidad de experimentar sensaciones nuevas e intensas, de estimulaciones fuertes, de conocerse y conocer las propias limitaciones, estas son características muy asociadas a la adolescencia y a los aspectos ya mencionados, por esto a la hora explicar el consumo, la falta de dominio de habilidades sociales y autocontrol para el presente estudio se mostraron como factor de riesgo. (Folkman y Lazarus, 2015).

\section{Declaración de financiamiento y de conflictos de interés:}

El estudio fue financiado por los autores, quienes declaran no tener algún tipo de conflicto de interés en la investigación realizada.

\section{Correspondencia}

Ismael Morales Ojeda

Correo electrónico:

ismaelmoralesojeda@gmail.com

\section{REFERENCIAS BIBLIOGRÁFICAS}

Grusec, J. y Hastings, P. (Eds.) (2016). Handbook of socialization: Theory and research. New york: The Guilford Press.

Rodrigo, M. J.; Máiquez, M. L.; García, M.; Mendoza, R.; Rubio, A.; Martínez, A. y Martín J. C. (2004). Relaciones padres-hijos y estilos de vida en la adolescencia. Psicothema, 16(2), 203-210.

Allen, J. y Hauser, S. (1996). Autonomy and relatedness in adolescent-family interactions as predictors of young adults' states of mind regarding attachment. Development and Psychopathology, 8(4), 793-809.

Salkovskis, P (1985). Loneliness among children and young adolescent. Developmental Psychology, 21(6), 1025-1031.

Martínez Arias, R.; Fernández Alba, A. y Salgado Ruiz, A. (2005). Patrones de ocio en los adolescentes de la comunidad de Madrid. Conductas de riesgo: de Tom Raider al botellón. Madrid, España: Consejo Económico y Social.

Pallarés , J y Feixa , C. (2000). Espacios e itinerarios para el ocio juvenil nocturno. Estudios de Juventud, (50), 23-41.

Bordignon, N. A. (2005). El desarrollo psicosocial de Eric Erikson. El diagrama epigenético del adulto. Rev Lasallista Investig, 2(2), 50-63.

Erikson, E. H. (1970). Identidad, juventud y crisis. Buenos Aires: Paidós.

Laespada, T.; Iraurgi, I. y Aróstegi, E. (2004). Factores de riesgo y protección frente al consumo de drogas: Hacia un modelo explicativo del consumo de drogas en jóvenes de la CAPV. Bilbao, España: Informe. Universidad de Deusto, Instituto Deusto de Drogodependencias.

Luengo , M.; Romero , E.; Gómez Fragüela, J.; Guerra López, A.; Lence Pereiro, M. (1998). La prevención del consumo de drogas y la conducta antisocial en la escuela: Análisis y evaluación de un programa. Santiago de Compostel: Universidad de Santiago de Compostel.

Secades, R.; Fernández, J. y González, A. (2003). El papel de la familia: Factores de riesgo para el consumo de drogas. Proyecto Hombre, 47, $27-$ 38.

Ministerio de Salud/OPS (2014). Encuesta Mundial de Tabaquismo en Jóvenes [documento online]. Recuperado de: http://www.msal.gob.ar/ent/ images/stories/vigilancia/pdf/2014-03_informegyts-jovenes.pdf 
Office of National Drug Control Policy (s/f). Informe sobre programas e iniciativas [pág. web]. Disponible en: https://www.ncjrs.gov/ondcppubs/ policy/strategy/sobre.html

De Vincenzi, A. y Bareilles, G. (2011). Promoción de la salud y prevención escolar del consumo de drogas en contextos de vulnerabilidad social. Educación Educadores, 14(3), 577-600.

Navarro, J. (2000). Factores de riesgo y protección de carácter social. Madrid: Ayuntamiento de Madrid, Área de Servicios Sociales, Departamento del Plan Municipal.

Benítez, A. N. (2010). Factores protectores y de riesgo social en relación al consumo de sustancias en adolescentes rurales y urbanos. Tesis de Grado. Mendoza: Universidad del Aconcagua.

Pedrón, V. (2008). Inicio temprano del consumo: ¿cómo medirlo? Anuario de Investigaciones, 15, 313-320.

López , K. S. (2008). Conducta antisocial y consumo de alcohol en adolescentes escolares. Rev Lat Am Enfermagem, 16(2), 299-305.
MSAL (2011). Algunos datos sobre el consumo de alcohol en Argentina. Buenos Aires, Argentina: MSAL.

Miotto Wright, M. G.; Gliksman, L.; Khenti, A. y Ferreira Furegato, R. (2009). Investigación sobre el fenómeno de las drogas bajo el abordaje de los estudios multicéntricos en América Latina y el Caribe. Rev Lat Am Enfermagem, 17, 759-61.

Fernández Hermida, J. R. y Secades Villa, R. (2008). Prevalencia del consumo de drogas y factores de riesgo y protección en población juvenil de 12 a 18 años de Asturias. Oviedo, España: Universidad de Oviedo.

Folkman, S. y Lazarus, R. (2015). Stress, appraisal and coping. New York: Springer.

Elzo, J.; Elorza, M. A. y Laespada, M. T. (1994). Alcoholismo juvenil: reflexiones y sugerencias de actuación ante una realidad contrastada. Bilbao: Universidad de Deusto, Instituto Deusto de Drogodependencias.

Recibido: $10 / 07 / 2019$

Aceptado: 12/04/2019 